\title{
COMUNICACIÓN
}

\section{Aspectos morfológicos de Paradeletrocephalus minor (Molin, 1861) Freitas \& Lent, 1947, en ñandu (Rhea americana), por medio de miscroscopio de luz y microscopio electrónico de barrido}

\author{
JAVIER ACOMOLLI*, DIEGO OCAYO**, ANTONIA C. SANTA CRUZ**, \\ FERNANDO MILANO*** y JUAN P. ROUX**
}

\begin{abstract}
MORPHOLOGYC ASPECTS OF Paradeletrocephalus minor (MOLIN, 1861) FREITAS \& LENT, 1947. IN ÑANDU (Rhea americana), BY LIGHT MICROSCOPE AND SEM
\end{abstract}

The aim of this study was to report some external morphological features of Paradeletrocephalus minor MOLIN (1861); FREITAS \& LENT (1947). The parasites were collected during necropsy from the small intestine and large intestine of Ñandú (Rhea americana), hunted in its native environment near Tandil City, Buenos Aires Province, Argentina. Collected specimens of Paradeletrocephalus minor were observed by light microscopy in order to be classified. Later the parasites were put into a $3 \%$ glutaraldehyde solution, dehydrated, dried by critical point, assembled, metalized, observed and photographed with a SEM. This is the first description of some external morphologic features of this species carried out by Light microscope and SEM in Argentina.

Key Words: Rhea americana, Nandu parasites, Paradeletrocephalus minor.

\section{INTRODUCCIÓN}

En la actualidad la cría en cautiverio del ñandú está emergiendo como una nueva alternativa en la industria de la carne, por estar considerada como la proteína del segundo milenio, consumida, producida y promocionada su producción en los países desarrollados. De las aves autóctonas es la más grande de Sudamérica y su área de distribución, en la
Argentina, comprende las regiones centro, norte y este. Su cría en cautiverio presenta diferencias con las de un animal tradicional por provenir del ambiente silvestre y no estar aun domesticados. Actualmente en el país se está promocionando su uso sustentable como recurso natural.

En los criaderos existentes en Venezuela, Brasil, Uruguay, Argentina y otros países limítrofes la oferta de producción todavía es reducida e insuficiente para satisfacer la demanda

\footnotetext{
* Becario de la Secretaria General de Ciencia y Técnica. Universidad Nacional del Nordeste. Cátedra de Introducción a las Ciencias Básicas, Sargento Cabral 2139 - (3400) Corrientes, Argentina. Tel. Fax: + 54 (3783) 425753. E-mail: jacomolli@vet.unne.edu.ar

** Docentes de la Cátedra de Introducción a las Ciencias Básicas, Sargento Cabral 2139 - (3400) Corrientes, Argentina. Tel. Fax: + 54 (3783) 425753. E-mail:

*** Docentes del Dpto. de Ciencias Básicas, Fac. de Ciencias Veterinarias de la Universidad Nacional del Centro de la Provincia de Buenos Aires.
} 
de países europeos y asiáticos, una de las limitaciones importantes que dificulta establecer las apropiadas pautas de manejo, es el insuficiente conocimiento de las enfermedades que los afectan tanto en vida silvestre como en cautividad.

Dentro del espectro de las enfermedades, se destacan como más frecuentes las parasitarias porque causan un gran impacto en los criaderos por su influencia negativa en la eficiencia y rentabilidad productiva, existiendo escasos reportes sobre ellas ${ }^{1}$. En nuestro país no se conocen citas de las parasitosis en estas aves, si bien son animales resistentes en condiciones de libertad o cría extensivas, debemos recordar que las producciones que tienden a la intensificación, deben afrontar problemas inherentes al hacinamiento que sufren los animales, y un gran inconveniente para los productores, en estos casos son las parasitosis. Dentro de estas debemos nombrar algunas en particular como la producida por nematodes del orden Strongylida (Diesing, 1851), que como sus parientes cercanos los pequeños strongylos (hematófagos), producen graves cuadros de decaimiento, diarreas y anemia $^{2}$.

El objetivo de éste trabajo fue describir algunos aspectos morfológicos externos de Paradeletrocephalus minor Molin (1861); Freitas \& Lent (1947), en Ñandú (Rhea americana), usando imágenes obtenidas por microscopio de luz y microscopio electrónico de barrido.

\section{MATERIAL Y MÉTODOS}

Se trabajó con 4 ejemplares (N1, N2, P1 y P2), provenientes de la estancia Los Planteles, ubicada a $35 \mathrm{~km}$ de la ciudad de Tandil, Provincia de Buenos Aires, Argentina. La densidad poblacional de estas aves fue de 130 animales, en un potrero de 60 ha, donde compartían el pastoreo con ganado bovino en engorde, las pasturas implantadas de ray grass, trébol rojo, pasto ovillo, cebadilla, trébol blanco, etc. Las aves fueron halladas muertas por causas desconocidas.

Tres de ellas (N1, N2 y P1) murieron a principios de septiembre, en estos casos el potrero tenía muy poca disponibilidad de pastura (menos de $1.000 \mathrm{~kg}$ de materia seca / ha), los animales tenían un mal estado de nutrición, se apreciaba un cuadro diarreico, estas heces eran muy líquidas.

El cuarto (P2) murió en diciembre, la disponibilidad forrajera era alta $(2.500 \mathrm{~kg}$ de $\mathrm{M}$ $\mathrm{S} / \mathrm{ha}$ ) y estaba en buen estado de nutrición.

A todos se les practicó la necropsia y se observó a todos los órganos de la economía, hallándose en el intestino delgado y grueso, numerosos nematodes que fueron colocados en frascos rotulados con solución de Railliet \& Henry como conservante, para su posterior estudio bajo microscopio de luz. Los helmintos destinados a la observación por microscopio electrónico de barrido (MEB) se sometieron a deshidratación en acetona con gradación creciente, secados a punto crítico, montados, metalizados en un baño de oro - paladio, observados y microfotografiados.

Para la identificación específica de los parásitos se utilizó las claves de Satyu Yamaguti (Sistema Helminthum, Part IV).

\section{RESULTADOS}

Se registraron datos de localización, número de parásitos hallados, sexo y caracteres morfométricos de los especimenes hallados (Tablas 1 y 2).

Los detalles morfológicos se pueden apreciar

Tabla 1. Distribución de P. minor por región del aparato gastrointestinal y por sexo. Medidas morfométricas en cm

\begin{tabular}{lcccrrr}
\hline Localización & Sexo & Animal N1 & Animal N2 & Animal P1 & Animal P2 & Media \\
\hline Intestino Delgado & A & 1,206 & & 1,16 & & 1,183 \\
\multirow{2}{*}{ Ciego } & B & 0,895 & & 1,16 & & 1,0275 \\
& A & 0,67 & 0,8 & 0,596 & 0,63 & 0,674 \\
Colon - Recto & B & 0,69 & 0,68 & 0,51 & 0,68 & 0,64 \\
& A & 0,86 & & 0,9 & 0,88 & 0,88 \\
& B & 0,865 & & 0,87 & 0,758 & 0,831 \\
\hline
\end{tabular}


Tabla 2. Total de $P$. minor recolectados, distribuidos por región del aparato gastrointestinal

\begin{tabular}{lcrcc}
\hline & Int. Delgado & Ciego & Colon - Recto & Total \\
\hline Animal N1 & 411 & 479 & 96 & 986 \\
Animal N2 & & 126 & & 126 \\
Animal P2 & & 61 & 720 & 781 \\
Animal P1 & 6 & 25 & 216 & 247 \\
\hline
\end{tabular}

en las fotos obtenidas por microscopio de luz y microscopio electrónico de barrido. La aplicación de la microscopía electrónica de barrido permitió la observación de los caracteres morfológicos superficiales no detectados por el anterior.

Los cuadros muestran la gran carga parasitaria de tres animales muertos, con un marcado descenso del número de parásitos en el cuarto animal. También se pudo determinar que la mayor cantidad de los mismos se encontraban parasitando el intestino grueso (ciegos, colon y recto) contrastando con el intestino delgado donde se pudo apreciar una sensible disminución de los mismos (Tabla 2).

\section{DISCUSIÓN}

La familia Deletrocephalidae comprende dos géneros Deletrocephalus y Paradeletrocephalus, el primero de ellos se caracteriza por poseer los elementos de la corona externa radiada estriada, la corona interna sinuosa, fondo de la cápsula bucal sin crestas y el esófago casi cilíndrico ${ }^{\mathbf{3}, 4}$; en cambio el segundo no presenta corona radiada

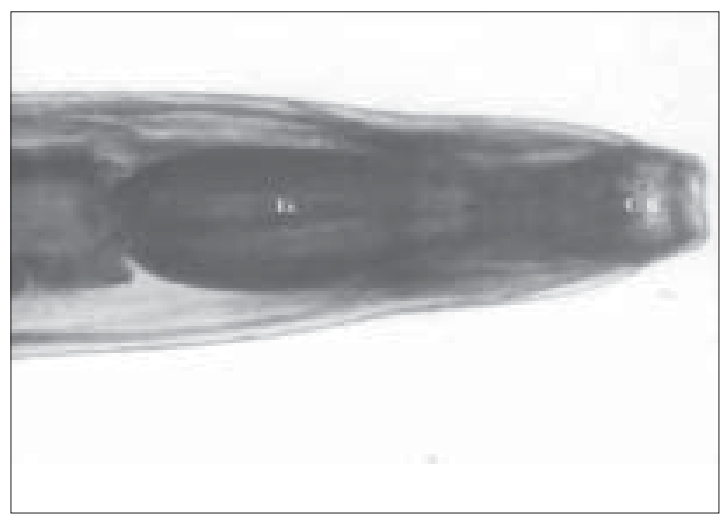

Figura 1. Paradeletrocephalus minor. Extremidad anterior. Microscopio de luz (4 x) Es: Esófago claviforme. C.B.: Cavidad bucal. externa, la corona radiada interna no es sinuosa, el fondo de la cápsula bucal presenta crestas verticales y el esófago es en forma de clava ${ }^{5}$. Figuras 1 y 2.

La hembra presenta una terminación del cuerpo abrupta con una punta afilada, la vulva se abre cerca del ano cubierta por una gran prominencia ${ }^{6}$. Figuras 3 y 4 . Huevos grandes, ovales, de aproximadamente $190-200 \mu \mathrm{m}$ de largo por 60 - $70 \mu \mathrm{m}$ de ancho, con una mórula ${ }^{6}$. Figuras 5 y 6.

El macho presenta una bolsa copulatriz abierta centralmente. Espícula filiforme, con alas, transversalmente estriado ${ }^{5}$. Figuras 7 y 8 .

Cabe destacar las diferencias morfométricas que se dan entre individuos en las distintas regiones del Aparato Gastrointestinal apreciable en la Tabla 1.

La especie descripta está acorde a la bibliografía consultada y responde a las características morfométricas de $P$. minor. Esta especie, que aparentemente se alimenta de sangre, puede ser una de las causas del síndrome anémico del ñandú, principalmente cuando en altas infestaciones ${ }^{1}$.

A través de este estudio los autores reportan por primera vez en Argentina algunos aspectos de la morfología externa que caracterizan a este helminto, mediante el uso del microscopio de luz y microscopio electrónico de barrido (MEB). Orden: Strongylida (Diesing, 1851) - Superfamilia: Strongyloidea - Familia: Deletrocephalidae (Freitas \& Lent, 1949), especie: P. minor.

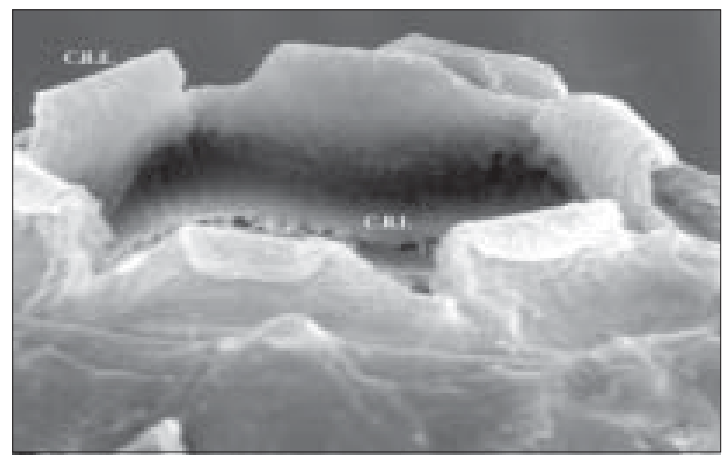

Figura 2. Paradeletrocephalus minor. Extremidad anterior. M.E.B.: (2.200 x). Diámetro del orificio bucal $52.3 \mu$ C.R.E.: Corona Radiada Externa, compuesta de 6 labios membranosos. C.R.I.: Corona Radiada Interna, elementos denticulares. 


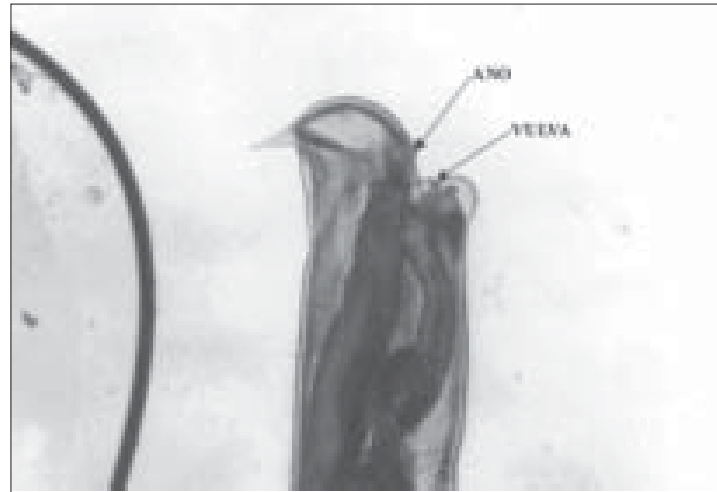

Figura 3. Paradeletrocephalus minor. Extremidad posterior hembra microscopio de luz $(4 \mathrm{x})$.

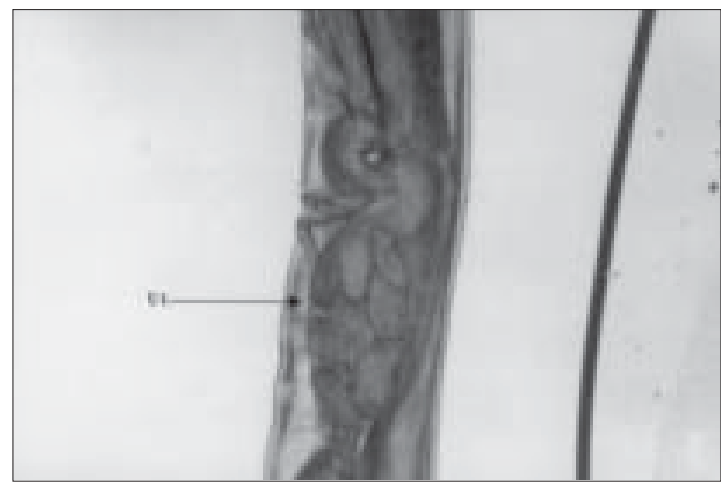

Figura 5. Paradeletrocephalus minor. Hembra microscopio de luz (4 x). U.: Útero con huevos.

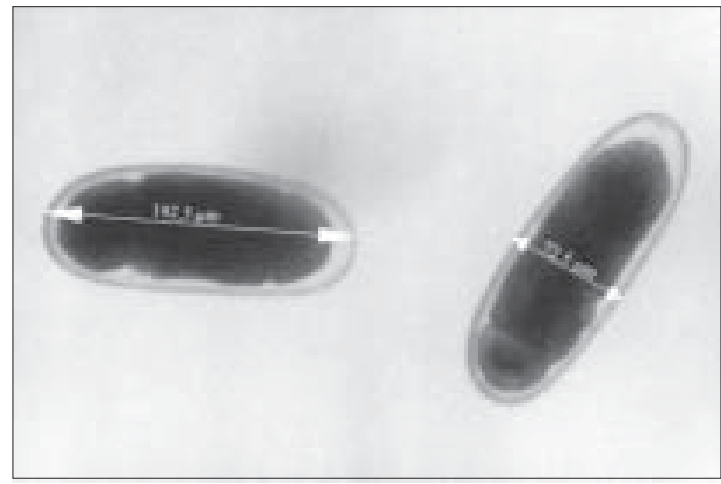

Figura 7. Paradeletrocephalus minor. Extremidad posterior macho. Microscopio de luz (4 x). Esp.: Espícula con alas laterales.

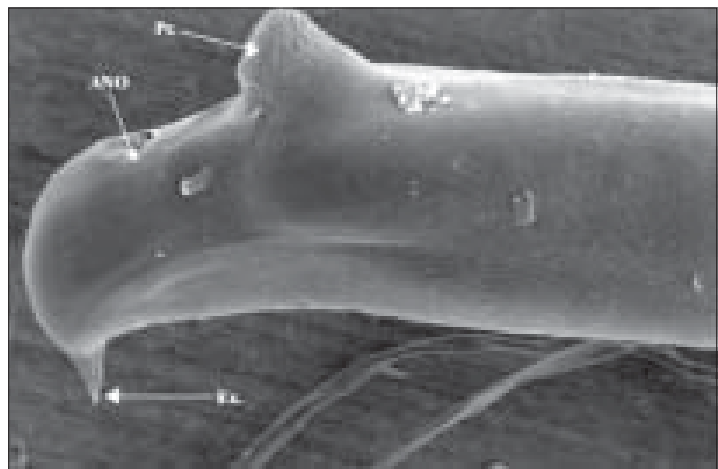

Figura 4. Paradeletrocephalus minor. Extremidad posterior hembra M.E.B.: $(280$ x). Ex.: Extremidad terminada en punta. Pr.: Prominencia que cubre a la vulva.

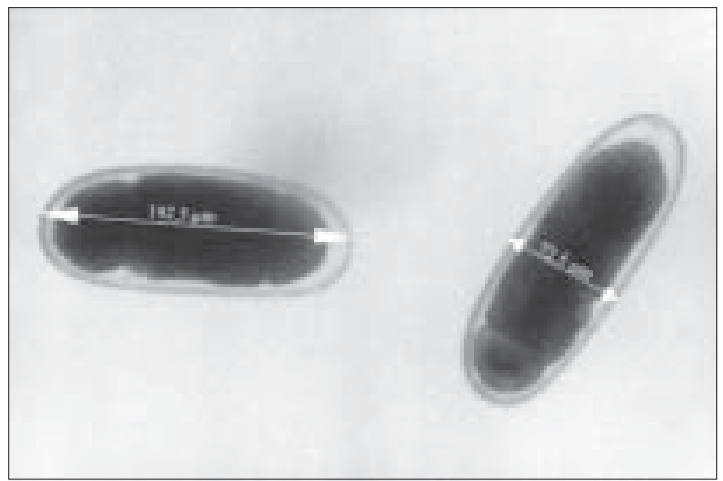

Figura 6. Paradeletrocephalus minor. Huevos morulados. Microscopio de luz (10 x).

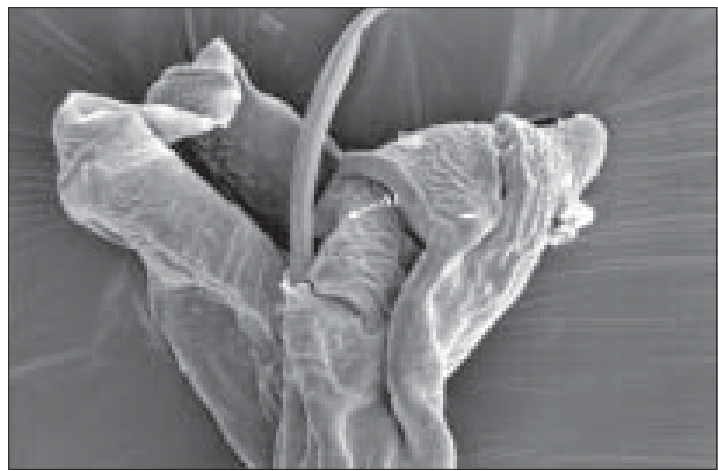

Figura 8. Paradeletrocephalus minor. Extremidad posterior macho. M.E.B.:(220 x). Bolsa copulatriz. Esp.: Espícula. Al.: Alas laterales. Longitud $269 \mu$. 


\section{REFERENCIAS}

1.- ZETTERMANN C D. Helmintos parásitos de Emas (Rhea americana) do Pantanal do Mato Grosso do Sul e de Jaboticabal. Dissertação (Mestre). 2000; 49 pp.

2.- SOULSBY E J L. Helminths, Artropods and Protozoa of Domesticated Animals. $7^{\text {th }}$ Edition. 809pp. bailliere Tindall, London, UK. 1982.

3.- DUNN A M. Helmintología Veterinaria. Manual Moderno. 1983; 389 pp.
4.- EWING M, YONZON M, PAGE R, et al. Delectrocephalus dimidiatus infestation in an adult Rhea (Pterocnemia pennata). Avian Dis 1995; 39: 441 -3.

5.- MASI PALLARES R. Nematodes de los Vertebrados de Paraguay y Otros Parásitos del Nuevo Mundo. Instituto de Investigaciones en Ciencias de la Salud. Universidad Nacional de Asunción, Paraguay. 1990; $348 \mathrm{pp}$.

6.- YAMAGUTI S. Systema Helminthum Vol. III, The Nematodes of Vertebrates. New York, Interscience Publisher, Inc. 1961; Part I: 679. Part II: 692. 